\title{
Cryptanalysis of $2 \mathrm{R}^{-}$Schemes
}

\author{
Jean-Charles Faugère $^{1}$ and Ludovic Perret $^{2}$ \\ ${ }^{1}$ LIP6, 8 rue du Capitaine Scott, Paris 75015, France \\ Jean-Charles.Faugere@inria.fr \\ ${ }^{2}$ UCL, Crypto Group, Microelectronic Laboratory \\ Place du Levant, 3 \\ Louvain-la-Neuve, B 1348, Belgium \\ ludovic.perret@uclouvain.be
}

\begin{abstract}
In this paper, we study the security of $2 \mathrm{R}^{-}$schemes 17$] 18$, which are the "minus variant" of two-round schemes. This variant consists in removing some of the $n$ polynomials of the public key, and permits to thwart an attack described at Crypto'99 25] against two-round schemes. Usually, the "minus variant" leads to a real strengthening of the considered schemes. We show here that this is actually not true for $2 \mathrm{R}^{-}$ schemes. We indeed propose an efficient algorithm for decomposing $2 \mathrm{R}^{-}$ schemes. For instance, we can remove up to $\left\lfloor\frac{n}{2}\right\rfloor$ equations and still be able to recover a decomposition in $O\left(n^{12}\right)$. We provide experimental results illustrating the efficiency of our approach. In practice, we have been able to decompose $2 \mathrm{R}^{-}$schemes in less than a handful of hours for most of the challenges proposed by the designers [18. We believe that this result makes the principle of two-round schemes, including $2 \mathrm{R}^{-}$schemes, useless.
\end{abstract}

Keywords: Cryptanalysis, Functional Decomposition Problem (FDP), Gröbner bases, $\mathrm{F}_{5}$ algorithm.

\section{Introduction}

Last years a new kind of cryptanalysis has made its entrance in cryptography: the so-called algebraic cryptanalysis. A fundamental issue of this cryptanalysis consists in finding zeroes of algebraic systems. Gröbner bases, which are a fundamental tool of commutative algebra, constitute the most elegant and efficient way for solving this problem. They provide an algorithmic solution for solving several problems related to algebraic systems (some of them can be found in [1]). We present here a new application of Gröbner bases. More precisely, we propose a new algorithm for solving the Functional Decomposition Problem (FDP). The problem is as follows:

\section{Functional Decomposition Problem (FDP)}

Input : multivariate polynomials $h_{1}, \ldots, h_{u}$.

Find : - if any - multivariate polynomials $f_{1}, \ldots, f_{u}$, and $g_{1}, \ldots, g_{n}$, such that:

$$
\left(h_{1}, \ldots, h_{u}\right)=\left(f_{1}\left(g_{1}, \ldots, g_{n}\right), \ldots, f_{u}\left(g_{1}, \ldots, g_{n}\right)\right) .
$$

This problem is related to security of $2 \mathrm{R}^{-}$schemes $17[18$.

C. Dwork (Ed.): CRYPTO 2006, LNCS 4117, pp. 357-372 2006.

(C) International Association for Cryptologic Research 2006 


\subsection{Related Works}

As stated by E. Biham [6], "the design of this scheme (2R) is unique as it uses techniques from symmetric ciphers in designing public key cryptosystems, while still claiming security based on relation to the difficulty of decomposing compositions of multivariate ... functions". Anyway, the security of $2 \mathrm{R}$ schemes has been already carefully investigated [6 25 26]. E. Biham proposed in [6] a successful cryptanalysis of $2 \mathrm{R}$ schemes with S-Boxes. This attack exploits the birthday paradox, but can be avoided by increasing the security parameters of $2 \mathrm{R}$ schemes [18]. At Crypto'99 [25], D.F. Ye, Z.D. Dai, and K.Y. Lam have presented a quite efficient method for solving the Functional Decomposition Problem. The security of $2 \mathrm{R}$ schemes is indeed related to this problem. To thwart this last attack, L. Goubin and J. Patarin have proposed [18] to use a general technique for repairing multivariate schemes, namely keeping secret some polynomials of the public key. The resulting schemes are called $2 \mathrm{R}^{-}$schemes. Note that V. Carlier, H. Chabanne, and E. Dottax [9] have described a method for protecting the confidentiality of block ciphers design exploiting the principle of $2 \mathrm{R}^{-}$schemes. Usually, the "minus modification" leads to a real strengthening of the considered schemes. For instance, $C^{*}$ is broken [22] while $C^{*--}$ is the basis of Sflash [10], the signature scheme recommended for low-cost smart cards by the European consortium Nessie1. Here, we show that $2 \mathrm{R}^{-}$is not more secure than $2 \mathrm{R}$.

\subsection{Organization of the Paper and Main Results}

The paper is organized as follows. We begin in Section 2 by introducing our notations and defining essential tools used in this paper, namely ideals, Gröbner bases, and several operations on ideals (sum, intersection, quotient, ... ). Section 3 gives a brief review of one-round, $2 \mathrm{R}$ and $2 \mathrm{R}^{-}$schemes. We also present the Functional Decomposition problem (FDP) in a more formal manner, which is at the basis of the security of $2 \mathrm{R}$ and $2 \mathrm{R}^{-}$schemes. An algorithm for solving this problem efficiently would allow to decompose the public key of $2 \mathrm{R}$ and $2 \mathrm{R}^{-}$ schemes into two independent quadratic systems, making thereby the principle of these cryptosystems useless. In Section 4, we present a general algorithm for solving FDP. Our method is inspired on the algorithm of D.F. Ye, Z.D. Dai, and K.Y. Lam [25]. Note that their algorithm only works for particular instances of FDP, namely when $u=n$, or $u=n-1$. Briefly, our algorithm works as follows. Let $\left(h_{1}, \ldots, h_{u}\right)=\left(f_{1}\left(g_{1}, \ldots, g_{n}\right), \ldots, f_{u}\left(g_{1}, \ldots, g_{n}\right)\right)$ be an instance of FDP. We first construct the ideal $\partial \mathcal{I}_{h}=\left\langle\frac{\partial h_{i}}{\partial x_{j}}: 1 \leq i \leq u, 1 \leq j \leq n\right\rangle$ generated by the partial derivatives of the $h_{i}$ s. We then show that for all $i, 1 \leq i \leq n, x_{n}^{d+1} g_{i} \in \partial \mathcal{I}_{h}$, for some $d \geq 0$. In most cases, this allows to recover a basis of the vector space $\mathcal{L}(g)=\operatorname{Vect}\left(g_{1}, \ldots, g_{n}\right)$ generated by $g_{1}, \ldots, g_{n}$. This is the most difficult part of our algorithm. The $f_{i}$ s being indeed recovered from the knowledge of $\mathcal{L}(g)$ by solving a linear system. The complexity of this algorithm depends on the ratio $n / u$. For example, our algorithm runs in $O\left(n^{12}\right)$, if $n / u<1 / 2$. More generally,

\footnotetext{
${ }^{1}$ https://www. cosic.esat.kuleuven.be/nessie/deliverables/decision-final.pdf
} 
we provide a global analysis of the theoretical complexity of our method. As a side effect, we give several insights into the theoretical behavior of the algorithm of D.F. Ye, Z.D. Dai, and K.Y. Lam. We conclude this section by providing experimental results illustrating the efficiency of our approach. We have been able to solve in few hours instances of FDP used in $2 \mathrm{R}^{-}$schemes for most of the challenges proposed in [18].

\section{Preliminaries}

Throughout this paper, we denote by $\mathbb{K}\left[x_{1}, \ldots, x_{n}\right]$ the polynomial ring in the $n$ indeterminate $x_{1}, \ldots, x_{n}$ over a finite field $\mathbb{K}$ with $q=p^{r}$ elements ( $p$ a prime, and $r \geq 1)$. The set of polynomials $p_{1}, \ldots, p_{s}$ of $\mathbb{K}\left[x_{1}, \ldots, x_{n}\right]$ can be regarded as a mapping $\mathbb{K}^{n} \rightarrow \mathbb{K}^{s}$ :

$$
\left(v_{1}, \ldots, v_{n}\right) \mapsto\left(p_{1}\left(v_{1}, \ldots, v_{n}\right), \ldots, p_{s}\left(v_{1}, \ldots, v_{n}\right)\right) .
$$

We will call these polynomials components. We will also denote by $\mathcal{I}=\left\langle p_{1}, \ldots, p_{s}\right\rangle$ $=\left\{\sum_{k=1}^{s} p_{k} u_{k}: u_{1}, \ldots, u_{s} \in \mathbb{K}\left[x_{1}, \ldots, x_{n}\right]\right\}$ the ideal generated by $p_{1}, \ldots, p_{s}$. We define now essential notions used in this paper. For a more thorough introduction to these tools, we refer to classical books on commutative algebra, such as [11]. Most of the results presented in this part are well known in commutative algebra, and thus given without proofs. For these proofs, we also refer to [11]. The reader already familiar with Gröbner bases and quotient ideals can skip this part.

\subsection{Gröbner Bases}

Informally, a Gröbner basis of an ideal is a generatring set of this ideal with "good" algorithmic properties. These bases are defined with respect to monomial orders. Here, we will use the lexicographic (LEX) and degree reverse lexicographical (DRL) orders, which are definedas follows:

Definition 1. Let $\alpha=\left(\alpha_{1}, \ldots, \alpha_{n}\right)$ and $\beta=\left(\beta_{1}, \ldots, \beta_{n}\right) \in \mathbb{N}^{n}$. Then: $-x_{1}^{\alpha_{1}} \cdots x_{n}^{\alpha_{n}} \prec_{L E X} x_{1}^{\beta_{1}} \cdots x_{n}^{\beta_{n}}$, if the left-most nonzero entry of the vector $\alpha-\beta$ is positive.

- $x_{1}^{\alpha_{1}} \cdots x_{n}^{\alpha_{n}} \prec_{D R L} x_{1}^{\beta_{1}} \cdots x_{n}^{\beta_{n}}$, if $\sum_{i=1}^{n} \alpha_{i}>\sum_{i=1}^{n} \beta_{i}$, or $\sum_{i=1}^{n} \alpha_{i}=\sum_{i=1}^{n} \beta_{i}$ and the right-most nonzero entry of $\alpha-\beta$ is negative.

To define Gröbner bases, we have to introduce the following definitions.

Definition 2. For any $n$-uple $\alpha=\left(\alpha_{1}, \ldots, \alpha_{n}\right) \in \mathbb{N}^{n}$, we denote by $x^{\alpha}$ the monomial $x_{1}^{\alpha_{1}} \cdots x_{n}^{\alpha_{n}}$. We define the total degree of this monomial by the sum $\sum_{i=1}^{n} \alpha_{i}$. The leading monomial of a polynomial $f \in \mathbb{K}\left[x_{1}, \ldots, x_{n}\right]$ is the largest monomial - w.r.t some monomial ordering $\prec-$ among the monomials of $f$. This leading monomial will be denoted by $\operatorname{LM}(f, \prec)$. The leading coefficient of $f$, denoted by $\mathrm{LC}(f, \prec)$, is the coefficient of $\operatorname{LM}(f, \prec)$ in $f$. The degree of $f$ - denoted $\operatorname{deg}(f)$ - is the total degree of $\operatorname{LM}(f, \prec)$. Finally, the maximal total degree of $f$ is the maximal total degree of the monomials occurring in $f$. 
We are now ready to define one of the main objects of this paper. Indeed:

Definition 3. A set of polynomials $G$ is a Gröbner basis - w.r.t. a monomial ordering $\prec-$ of an ideal $\mathcal{I}$ in $\mathbb{K}\left[x_{1}, \ldots, x_{n}\right]$, if for all $f \in \mathcal{I}$ there exists $g \in G$ such that $\operatorname{LM}(g, \prec)$ divides $\operatorname{LM}(f, \prec)$. This Gröbner basis is called reduced if, for all $g \in G, \mathrm{LC}(g, \prec)=1$, and any monomial of $g \in G$ is not divisible by any element of $\operatorname{LM}(G \backslash\{g\}, \prec)$. Let $G$ be Gröbner basis - w.r.t. a monomial ordering $\prec-$ of an ideal $\mathcal{I}$ in $\mathbb{K}\left[x_{1}, \ldots, x_{n}\right]$, and $d$ be a positive integer. We call $d$-Gröbner basis (or truncated Gröbner basis) of an homogeneous ideal $\mathcal{I}$ the set:

$$
\{g \in G: \operatorname{deg}(g)=d\}
$$

A Gröbner basis of a given ideal is not unique in general. The reduced Gröbner basis allows to achieve uniqueness. A reduced Gröbner basis can be obtained from a Gröbner basis in polynomial-time. Gröbner bases are a fundamental tool to study algebraic systems in theory and practice. They provide an algorithmic solution for solving several problems related to polynomial systems (some of them can be found in [1]). The historical method for computing Gröbner bases is Buchberger's algorithm [87]. Recently, more efficient algorithms have been proposed. To date, $\mathrm{F}_{5}[13$ is the most efficient for computing Gröbner bases (a brief description of this algorithm is given in Appendix A). Here we will concentrate on Gröbner bases w.r.t. lexicographical and degree reverse lexicographical orders.

\section{LEX and DRL Gröbner Bases}

Lexicographical Gröbner bases (LEX Gröbner bases) offer a way for eliminating variables.

Theorem 1 (Elimination Theorem). Let $\mathcal{I}$ be an ideal in $\mathbb{K}\left[x_{1}, \ldots, x_{n}\right]$, and $k \in\{1, \ldots, n\}$. If $G$ is a LEX Gröbner basis of $\mathcal{I}$, then $G \cap \mathbb{K}\left[x_{k+1}, \ldots, x_{n}\right]$ is a Gröbner basis of $\mathcal{I} \cap \mathbb{K}\left[x_{k+1}, \ldots, x_{n}\right]$.

The shape of degree reverse lexicographical Gröbner bases (DRL Gröbner bases) is much more complicated. However, DRL Gröbner bases have several interesting properties. For instance, the polynomials of lowest degree of an ideal $\mathcal{I}$ appear in a DRL Gröbner bases of this ideal. More precisely:

Theorem 2. Let $\mathcal{I} \subset \mathbb{K}\left[x_{1}, \ldots, x_{n}\right], d=\min \{\operatorname{deg}(f): f \in \mathcal{I}\}$, and $G$ be a $D R L$ Gröbner basis of $\mathcal{I}$. Then:

$$
\operatorname{Vect}(g \in G: \operatorname{deg}(g)=d)=\operatorname{Vect}(g \in \mathcal{I}: \operatorname{deg}(g)=d) \text {. }
$$

Proof. A proof of this theorem can be found in [3].

We should mention that the variable $x_{n}$ has a special role for the DRL order.

Lemma 1. Let $f \in \mathbb{K}\left[x_{1}, \ldots, x_{n}\right]$, and $m$ be a positive integer. Then:

$$
x_{n}^{m}\left|f \Longleftrightarrow x_{n}^{m}\right| \mathrm{LM}(f, \prec D R L) .
$$




\section{Sum, Intersection, and Quotient of Ideals}

We now go over the definitions of several operations on ideals.

Definition 4. Let $\mathcal{I}$ and $\mathcal{J}$ be ideals in $\mathbb{K}\left[x_{1}, \ldots, x_{n}\right]$. Then:

- the sum of $\mathcal{I}$ and $\mathcal{J}$, noted $\mathcal{I}+\mathcal{J}$, is the $\mathcal{I}+\mathcal{J}=\{f+g: f \in \mathcal{I}$ and $g \in \mathcal{J}\}$. - the intersection of $\mathcal{I}$ and $\mathcal{J}$, is defined as $\mathcal{I} \cap \mathcal{J}=\left\{f \in \mathbb{K}\left[x_{1}, \ldots, x_{n}\right]: f \in\right.$ $\mathcal{I}$ and $f \in \mathcal{J}\}$.

$-\mathcal{I}+\mathcal{J}$ and $\mathcal{I} \cap \mathcal{J}$ are ideals.

Given two ideals and their generators, we would like to compute a set of generators for the intersection. This is actually much more delicate than the analogous problem for sums, which is straightforward. Indeed, $\mathcal{I}=\left\langle p_{1}, \ldots, p_{s}\right\rangle+\mathcal{J}=$ $\left\langle g_{1}, \ldots, g_{r}\right\rangle=\left\langle p_{1}, \ldots, p_{s}, g_{1}, \ldots, g_{r}\right\rangle$. The following result permits to solve the problem for intersections.

Theorem 3. Let $\mathcal{I}, \mathcal{J}$ be ideals in $\mathbb{K}\left[x_{1}, \ldots, x_{n}\right]$, and $t$ be a new variable. Then:

$$
\mathcal{I} \cap \mathcal{J}=(t \cdot \mathcal{I}+(1-t) \cdot \mathcal{J}) \cap \mathbb{K}\left[x_{1}, \ldots, x_{n}\right],
$$

where $t \cdot \mathcal{I}=\{t \cdot h: h \in \mathcal{I}\}$, and $(1-t) \cdot \mathcal{J}=\{(1-t) \cdot h: h \in \mathcal{J}\}$ are in $\mathbb{K}\left[t, x_{1}, \ldots, x_{n}\right]$.

This result, together with the Elimination Theorem (i.e. Theorem 1), provide a method for computing intersections of ideals. Given ideals $\mathcal{I}=\left\langle p_{1}, \ldots, p_{s}\right\rangle$ and $\mathcal{J}=\left\langle g_{1}, \ldots, g_{r}\right\rangle$ in $\mathbb{K}\left[x_{1}, \ldots, x_{n}\right]$, we consider the ideal $\left\langle t \cdot p_{1}, \ldots, t \cdot p_{s},(1-t)\right.$. $\left.g_{1}, \ldots,(1-t) \cdot g_{r}\right\rangle \subset \mathbb{K}\left[t, x_{1}, \ldots, x_{n}\right]$. Those elements of a LEX Gröbner basis (with $t \succ_{L E X} x_{1} \succ_{L E X} \cdots \succ_{L E X} x_{n}$ ) that do not contain the variable $t$ will exactly form a Gröbner basis for $\mathcal{I} \cap \mathcal{J}$.

Definition 5. Let $\mathcal{I}$ and $\mathcal{J}$ be ideals in $\mathbb{K}\left[x_{1}, \ldots, x_{n}\right]$. The ideal quotient of $\mathcal{I}$ by $\mathcal{J}$, denoted $\mathcal{I}: \mathcal{J}$, is the set

$$
\mathcal{I}: \mathcal{J}=\left\{f \in \mathbb{K}\left[x_{1}, \ldots, x_{n}\right]: f g \in \mathcal{I}, \text { for all } g \in \mathcal{J}\right\} .
$$

The following proposition relates the quotient operation to the sum and intersection operations.

Proposition 1. Let $\mathcal{I}$, and $\left\{\mathcal{I}_{k}\right\}_{1 \leq k \leq r}$ be ideals in $\mathbb{K}\left[x_{1}, \ldots, x_{n}\right]$. Then:

$$
\begin{aligned}
& \text { i) }\left(\bigcap_{k=1}^{r} \mathcal{I}_{k}\right): \mathcal{I}=\bigcap_{k=1}^{r}\left(\mathcal{I}_{k}: \mathcal{I}\right) \\
& \text { ii) } \mathcal{I}:\left(\sum_{k=1}^{r} \mathcal{I}_{k}\right)=\bigcap_{k=1}^{r}\left(\mathcal{I}: \mathcal{I}_{k}\right)
\end{aligned}
$$

If $f$ is a polynomial and $\mathcal{I}$ an ideal, we shall write $\mathcal{I}: f$ instead of $\mathcal{I}:\langle f\rangle$. A special case of ii) is:

$$
\mathcal{I}:\left\langle f_{1}, \ldots, f_{r}\right\rangle=\bigcap_{k=1}^{r}\left(\mathcal{I}: f_{k}\right) .
$$

We now address the question of computing generators of the ideal quotient $\mathcal{I}: \mathcal{J}$. The following observation is crucial: 
Theorem 4. Let $\mathcal{I}$ be an ideal in $\mathbb{K}\left[x_{1}, \ldots, x_{n}\right]$, and $f \in \mathbb{K}\left[x_{1}, \ldots, x_{n}\right]$. If $\left\langle g_{1}, \ldots, g_{p}\right\rangle=\mathcal{I} \cap\langle f\rangle$, then

$$
\left\langle g_{1} / f, \ldots, g_{p} / f\right\rangle=\mathcal{I}: f .
$$

In order to construct a basis of an ideal quotient, we proceed as follows. Given ideals $\mathcal{I}=\left\langle p_{1}, \ldots, p_{s}\right\rangle$ and $\mathcal{J}=\left\langle g_{1}, \ldots, g_{r}\right\rangle$ in $\mathbb{K}\left[x_{1}, \ldots, x_{n}\right]$, we compute a basis for the intersections $\mathcal{I} \cap\left\langle g_{1}\right\rangle, \ldots, \mathcal{I} \cap\left\langle g_{n}\right\rangle$ by using the above described method. For each $i$, we divide by $f$ each element of a basis of $\mathcal{I} \cap\left\langle g_{i}\right\rangle$. This leads to a basis for $\mathcal{I}: g_{i}$. We then obtain a basis for $\mathcal{I}: \mathcal{J}$ by computing the intersections $\bigcap_{k=1}^{r}\left(\mathcal{I}: g_{i}\right)$.

\section{$3 \quad 2 \mathrm{R}^{-}$Schemes}

In [20], T. Matsumoto and H. Imai proposed one of the first examples of PKCs using compositions of multivariate polynomials. The public key of one of them, called $\mathrm{C}^{*}([21])$, represented by " $t \circ \psi \circ s$ ", where $t, s$ are two secret linear mappings over $G F(2)^{n}$, and $\psi$ is the multivariate representation of $G F\left(2^{n}\right) \rightarrow$ $G F\left(2^{n}\right), x \mapsto x^{1+2^{\theta}}$. This scheme has been broken by J. Patarin at Crypto'95 22 .

One-round schemes [17/18 are generalizations of $\mathrm{C}^{*}$. The public key of these schemes is indeed of the form " $t \circ \psi \circ s$ ", where $t, s$ are two affine mappings over $\mathbb{K}^{n}$, and a $\psi: \mathbb{K}^{n} \rightarrow \mathbb{K}^{n}$ is a bijective mapping given by $n$ multivariate polynomials of degree two. J. Patarin and L. Goubin [17/8] propose several constructions for $\psi$ :

1. S-box functions: $\left(a_{1}, \ldots, a_{n}\right) \mapsto$

$$
\left(S_{1}\left(a_{1} \ldots, a_{n_{1}}\right), S_{2}\left(a_{n_{1}+1} \ldots, a_{n_{1}+n_{2}}\right), \ldots, S_{b}\left(a_{n_{1}+n_{2}+\cdots+n_{d-1}+1}, \ldots, a_{n}\right)\right),
$$

where $n=\sum_{i} n_{i}$, and each $S_{i}: K^{n_{i}} \rightarrow K^{n_{i}}$ is quadratic.

2. Triangular functions:

$$
\left(a_{1}, \ldots, a_{n}\right) \mapsto\left(a_{1}, a_{2}+q_{1}\left(a_{1}\right), a_{3}+q_{2}\left(a_{1}, a_{2}, a_{3}\right), \ldots, a_{n}+q_{n-1}\left(a_{1}, \ldots, a_{n-1}\right)\right),
$$

where each $q_{i}$ is quadratic.

3. Combinations of S-box and triangular functions.

They showed that all these constructions are insecure [17 18. To circumvent attacks, they introduce two-round schemes whose public key is the composition of two one-round schemes. The secret key of two-round schemes consists of:

Three affine bijections $r, s, t: K^{n} \rightarrow K^{n}$.

Two applications $\psi, \phi: K^{n} \rightarrow K^{n}$, given by $n$ quadratic polynomials.

The public key is composed of $n$ polynomials $p_{1}, \ldots, p_{n}$ of total degree 4 describing:

$$
p=t \circ \psi \circ s \circ \phi \circ r, K^{n} \rightarrow K^{n} .
$$


When all the polynomials are given, this scheme is called $2 R$ scheme. If only some of them are given, it is called $2 R^{-}$scheme. The public-key part of the computation is merely an application of the mapping $p$ (for encrypting a message, or checking the validity of a signature). For the secret-key computations, we need to invert the mappings $\psi$ and $\phi$. The authors then propose to choose the mappings among the constructions 1,2,3 described above and also:

4. $\mathrm{C}^{*}$ functions: monomials over an extension of degree $n$ over $K$,

5. $\mathrm{D}^{*}$ functions 16 .

In 17 18, it has been proved that when $\psi$ is chosen in the classes 2 . and 4 ., then the resulting $2 \mathrm{R}$ scheme is weak. It is not clear that a similar result holds for $2 \mathrm{R}^{-}$schemes.

Anyway, does composing two weak one-round schemes leads to a secure scheme ? The answer is closely related to the difficulty of the following problem:

\section{Functional Decomposition Problem (FDP)}

Input : $h=\left(h_{1}, \ldots, h_{u}\right) \in \mathbb{K}\left[x_{1}, \ldots, x_{n}\right]^{u}$.

Find : - if any $-f=\left(f_{1}, \ldots, f_{u}\right) \neq h \in \mathbb{K}\left[x_{1}, \ldots, x_{n}\right]^{u}$, and $g=\left(g_{1}, \ldots, g_{n}\right) \in$ $\mathbb{K}\left[x_{1}, \ldots, x_{n}\right]^{n}$, such that:

$$
\left(h_{1}(x), \ldots, h_{u}(x)\right)=\left(f_{1}\left(g_{1}(x), \ldots, g_{n}(x)\right), \ldots, f_{u}\left(g_{1}(x), \ldots, g_{n}(x)\right)\right),
$$

noted $h(x)=(f \circ g)(x)$ hereafter, where $x=\left(x_{1}, \ldots, x_{n}\right)$.

During the last years several results have been obtained on the univariate polynomial decomposition area [152324]. However, multivariate decomposition problem has not been studied so much. Particular instances (multi-univariate,...) of FDP have been investigated in 2319. In 12, M. Dickerson provided several insights into the theoretical complexity of FDP. However, this kind of results solely guarantees the difficulty of the worst-case. In the cryptographic context, D.F. Ye, Z.D. Dai, and K.Y. Lam presented in 2526 a quite efficient method for solving instances of FDP used in 2R. Note that their method only works when $u=n$, or $u=n-1$ [26]. To the best of our knowledge, there exists no previously known algorithm for solving FDP when $u<n-1$. An efficient method for solving FDP in this case would permit to decompose $2 \mathrm{R}^{-}$schemes into two independent schemes given by quadratic polynomials. To break these schemes, we then would only have to solve two quadratic systems. As mentioned by J. Patarin and L. Goubin [18], this would make the principle of two-round schemes, including $2 \mathrm{R}^{-}$, useless.

\section{A General Algorithm for Solving FDP}

In this part, we present a new algorithm for solving FDP. Our approach is inspired on the works of D.F. Ye, Z.D. Dai, and K.Y. Lam 2526. According to these authors, we can restrict our attention to homogeneous instances of FDP [25]. The homogenization of a polynomial $p \in \mathbb{K}\left[x_{1}, \ldots, x_{n}\right]$, denoted $p^{*}$, 
is defined by $p^{*}\left(x_{0}, x_{1}, \ldots, x_{n}\right)=x_{0}^{\operatorname{deg}(p)} p\left(x_{1} / x_{0}, \ldots, x_{n} / x_{0}\right)$, where $x_{0}$ is a new variable. For any mapping $f: \mathbb{K}^{n} \rightarrow \mathbb{K}^{u}$, given by the polynomials $f_{1}, \ldots, f_{u}$, we define its homogenization by $f^{*}=\left(x_{0}^{\operatorname{deg}(f)}, f_{1}^{*}, \ldots, f_{n}^{*}\right)$. The dehomogenization of $f^{*}$ is then $f=\left(f_{1}^{*}\left(1, x_{1}, \ldots, x_{n}\right), \ldots, f_{n}^{*}\left(1, x_{1}, \ldots, x_{n}\right)\right)$. We have:

Lemma 2 ([25]). Let $f: \mathbb{K}^{n} \rightarrow \mathbb{K}^{u}$ and $g: \mathbb{K}^{n} \rightarrow \mathbb{K}^{n}$ be two mappings, then:

$$
(f \circ g)^{*}=f^{*} \circ g^{*} \text {. }
$$

Note 1. In [25], it is stated that this lemma is correct only if $\operatorname{deg}(f) \operatorname{deg}(g)>|K|$. We no longer need this condition over $\mathbb{K}\left[x_{1}, \ldots, x_{n}\right]$.

Thus, if we can decompose $h^{*}=f^{*} \circ g^{*}$, then a decomposition of $h=f \circ g$ is simply obtained by dehomogenization of $f^{*}$ and $g^{*}[25$. Now, we assume that $f: \mathbb{K}^{n} \rightarrow \mathbb{K}^{u}$ and $g: \mathbb{K}^{n} \rightarrow \mathbb{K}^{n}$ are two homogeneous functions of degree two. Finally, let $h=f \circ g$, and $\left\{h_{i}\right\}_{1 \leq i \leq u},\left\{f_{i}\right\}_{1 \leq i \leq u},\left\{g_{i}\right\}_{1 \leq i \leq n}$ be the components of $h, f, g$ respectively.

\subsection{Description of the Algorithm}

The aim of our algorithm is to find the vector space $\mathcal{L}(g)=\operatorname{Vect}\left(g_{1}, \ldots, g_{n}\right)$ generated by $g_{1}, \ldots, g_{n}$. More precisely, this vector space will be recovered from a DRL Gröbner basis of a suitable ideal. Note that the knowledge of $\mathcal{L}(g)$ is sufficient for decomposing $h$. Indeed, any bijective linear combination $A$ of the $g_{i}$ s leads to a decomposition of $h$ since:

$$
h=\left(f \circ A^{-1}\right) \circ(A \circ g) .
$$

Let us first assume that we know the vector space $\mathcal{L}(g)$. For all $i, 1 \leq i \leq u$ :

$$
\begin{aligned}
f_{i} & =\sum_{1 \leq k, \ell \leq n} f_{k, \ell}^{(i)} x_{k} x_{\ell} \in \mathbb{K}\left[x_{1}, \ldots, x_{n}\right], \\
g_{i} & =\sum_{1 \leq k, \ell \leq n} g_{k, \ell}^{(i)} x_{k} x_{\ell} \in \mathbb{K}\left[x_{1}, \ldots, x_{n}\right] .
\end{aligned}
$$

Therefore, for all $i, 1 \leq i \leq u$ :

$$
h_{i}=f_{i}\left(g_{1}, \ldots, g_{n}\right)=\sum_{1 \leq k, \ell \leq n} f_{k, \ell}^{(i)} g_{k} g_{\ell} .
$$

By comparing the coefficients in the right-most and left-most parts of these equalities, we obtain a linear system of $O\left(u \mathrm{C}_{n+2}^{2}\right)$ equations in the $u \mathrm{C}_{n+2}^{2}$ unknown coefficients of the $f_{i}$ s. It seems difficult to rigorously evaluate the rank of this linear system, a question that has been avoided in the previous works on FDP 25 26]. However, it is very likely that this linear system is of full rank when the $f_{i} \mathrm{~s}$ are dense polynomials. For the instances of FDP used in $2 \mathrm{R}^{-}$schemes, we experimentally only obtain linear systems of full rank. The difficult part is actually to determine the vector space $\mathcal{L}(g)$. For this, we observe that:

$$
\frac{\partial h_{i}}{\partial x_{j}}=\sum_{1 \leq k, \ell \leq n} f_{k, \ell}\left(\frac{\partial g_{k}}{\partial x_{j}} g_{\ell}+g_{k} \frac{\partial g_{\ell}}{\partial x_{j}}\right) \text {. }
$$


The polynomials $g_{1}, \ldots, g_{n}$ being of degree two, their partial derivatives are of degree one. Hence:

$$
\partial \mathcal{I}_{h}=\left\langle\frac{\partial h_{i}}{\partial x_{j}}: 1 \leq i \leq u, 1 \leq j \leq n\right\rangle \subseteq\left\langle x_{k} g_{\ell}\right\rangle_{1 \leq k, \ell \leq n}=\mathcal{V} .
$$

This ideal $\partial \mathcal{I}_{h}$ usually provides enough information for recovering the polynomials $g_{1}, \ldots, g_{n}$.

Theorem 5. Let $M(d)$ be the set of monomials of degree $d \geq 0$ in $x_{1}, \ldots, x_{n}$, and

$$
\begin{aligned}
& V_{d}=\operatorname{Vect}\left(m g_{k}: m \in M(d+1), \text { and } 1 \leq k \leq n\right), \\
& \tilde{V}_{d}=\operatorname{Vect}\left(\left\{m \frac{\partial h_{i}}{\partial x_{j}}: m \in M(d), 1 \leq i \leq u, \text { and } 1 \leq j \leq n\right\}\right) .
\end{aligned}
$$

Then, for all $i, 1 \leq i \leq n$ :

$$
x_{n}^{d+1} g_{i} \in \partial \mathcal{I}_{h}, \text { if } \operatorname{dim}\left(\tilde{V}_{d}\right) \geq n|M(d+1)|,
$$

where $\operatorname{dim}\left(\tilde{V}_{d}\right)$ is the dimension of $\tilde{V}_{d}$ as a vector space over $V_{d}$.

Proof. We first study the case $d=0$. Let $\tilde{V}=\tilde{V}_{0}$ be the linear space generated by the partial derivatives of the $h_{i}$ s, i.e.:

$$
\tilde{V}_{0}=\tilde{V}=\operatorname{Vect}\left(\left\{\frac{\partial h_{i}}{\partial x_{j}}\right\}_{1 \leq j \leq n}^{1 \leq i \leq u}\right) \subset \partial \mathcal{I}_{h} .
$$

According to (2), each element of $\tilde{V}$ can be written as a sum of $\left\{x_{k} g_{\ell}\right\}_{1 \leq k, \ell \leq n}$. Now let $A_{\tilde{V}} \in \mathcal{M}_{n^{2} \times n^{2}}(\mathbb{K})$ be a matrix associated to the linear transformation $\operatorname{Vect}\left(\left\{x_{k} g_{\ell}\right\}_{1 \leq k, \ell \leq n}\right) \mapsto \tilde{V}$. For some basis:

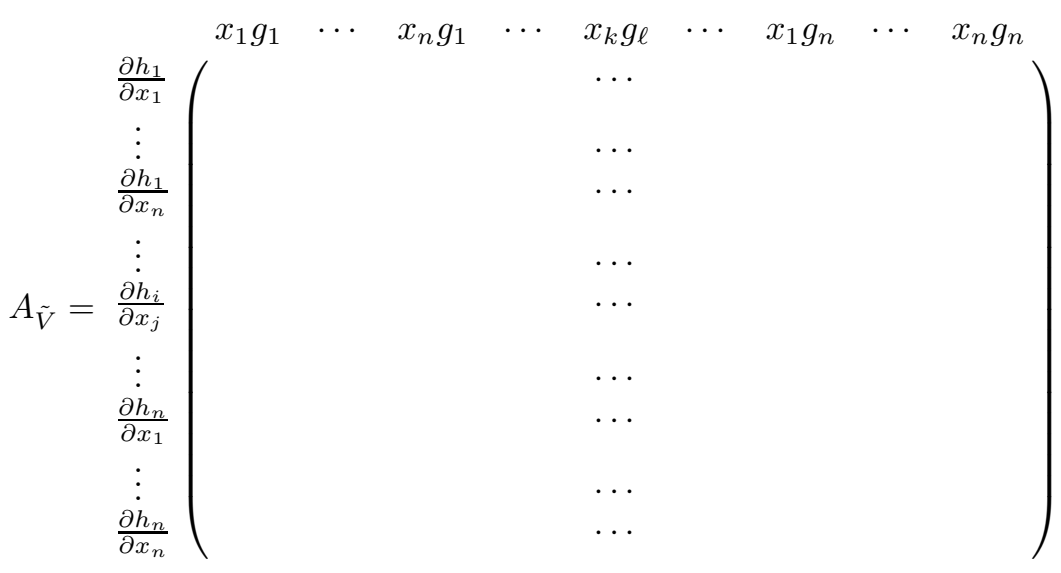

One can see at once that the $x_{n} g_{i}$ s lie in $\tilde{V}$ if the number of linearly independent rows of this matrix is at least equal to its number of columns. That is, $x_{n} g_{i} \in \partial \mathcal{I}_{h}$, for all $i, 1 \leq i \leq n$, if: 


$$
\operatorname{dim}(\tilde{V}) \geq n|M(1)|=n^{2} .
$$

Observe that $\operatorname{dim}(\tilde{V})$ is upper-bounded by un. Thus, $\operatorname{dim}(\tilde{V}) \geq n|M(1)|=n^{2}$ only holds if $u=n$. This explains why the method proposed in [25 26] is limited to $2 \mathrm{R}$ schemes. To circumvent this problem, we have to consider a vector space of higher dimension. This is the motivation for considering:

$$
\tilde{V}_{d}=\operatorname{Vect}\left(\left\{m \frac{\partial h_{i}}{\partial x_{j}}: m \in M(d), 1 \leq i \leq u, \text { and } 1 \leq j \leq n\right\}\right) .
$$

From (2), we deduce that each polynomial of $\tilde{V}_{d}$ can be written as a sum of elements of:

$$
V_{d}=\operatorname{Vect}\left(m g_{k}: m \in M(d+1), \text { and } 1 \leq k \leq n\right)
$$

Let then $A_{\tilde{V}_{d}}$ be a matrix associated to $V_{d} \mapsto \tilde{V}_{d}$. For some basis:

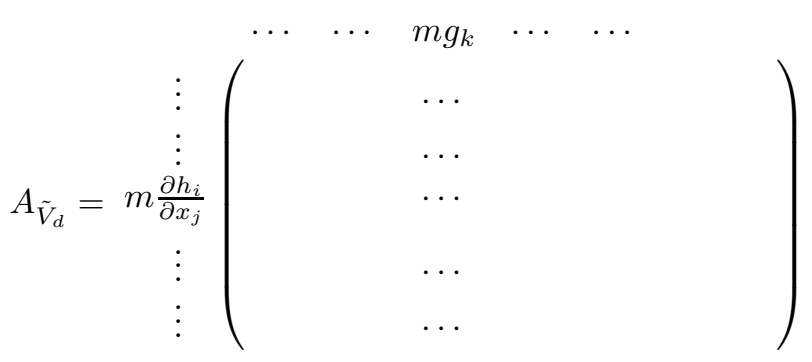

Thus, $x_{n}^{d+1} g_{i} \in \tilde{V} \subset \partial \mathcal{I}_{h}$, for all $i, 1 \leq i \leq n$, if $\operatorname{dim}\left(\tilde{V}_{d}\right)$ is at least equal to the number of columns of $A_{\tilde{V}_{d}}$. That is, if $\operatorname{dim}\left(\tilde{V}_{d}\right) \geq n|M(d+1)|$.

Remark 1. At the end of this part, we will provide an explicit value of $d$ in function of the ratio $n / u$.

According to Theorem [5, the polynomials $g_{i}$ s are contained, up to some power of $x_{n}$, in $\partial \mathcal{I}_{h}$. Therefore, the quotient of this ideal by a suitable power of $x_{n}$ contains the polynomials $g_{1}, \ldots, g_{n}$.

Corollary 1. Using the same notations as in Theorem 5, If $\operatorname{dim}\left(\tilde{V}_{d}\right) \geq n \mid M(d+$ 1)|, then:

$$
\mathcal{L}(g) \subset\left\langle g_{1}, \ldots, g_{n}\right\rangle \subseteq \partial \mathcal{I}_{h}:\left(x_{n}^{d+1}\right) .
$$

Proof. The proof of this corollary is obviously deduced from Theorem 5] and very definition of the quotient.

Thus each element of $\mathcal{L}(g)$ is included in $\partial \mathcal{I}_{h}:\left(x_{n}^{d+1}\right)$. Let then $G$ be a (reduced) DRL Gröbner basis of this ideal. It is then natural to consider the set $B_{g}=$ $\operatorname{Vect}(g \in G: \operatorname{deg}(g)=2)$, since according to Theorem 2 ,

$$
\mathcal{L}(g)=B_{g}, \text { if } \# B_{g}=n, \text { and } \min (\operatorname{deg}(g): g \in G)=2 .
$$


If these conditions are not fulfilled, then one can not recover efficiently $\mathcal{L}(g)$ from $B_{g}$. Observe that the condition \# $B_{g}=n$ implies that there exists a unique decomposition (up to bijective linear combinations). To get away with these problems, we can apply several heuristics such as computing $\partial \mathcal{I}_{h}:\left(x_{1}^{d+1}\right), \ldots, \partial \mathcal{I}_{h}$ : $\left(x_{n-1}^{d+1}\right)$. In practice, it has been always sufficient to compute $\partial \mathcal{I}_{h}:\left(x_{n}^{d}\right)$, for a suitable $d$ (i.e. $\left.\operatorname{dim}\left(\tilde{V}_{d}\right) \geq n|M(d+1)|\right)$.

\subsection{The Algorithm AlgofdP}

We describe now our algorithm for general instances of FDP, i.e. we no longer suppose here that $h$ is given by homogeneous polynomials.

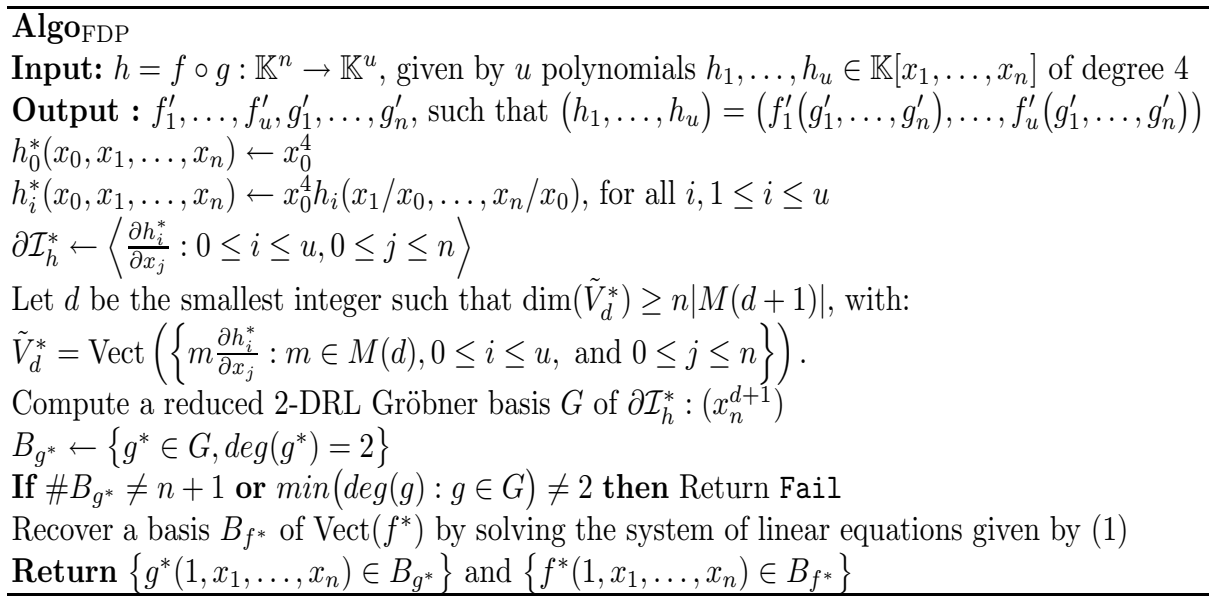

Remark 2. In practice, our algorithm never returned Fail for instances of FDP used in $2 R^{-}$.

Theorem 6. Let $g_{0}^{*}\left(x_{0}, x_{1}, \ldots, x_{n}\right)=x_{0}^{2}$, and $g_{i}^{*}\left(x_{0}, x_{1}, \ldots, x_{n}\right)=x_{0}^{2} g_{i}\left(x_{1} /\right.$ $\left.x_{0}, \ldots, x_{n} / x_{0}\right)$, for all $i, 1 \leq i \leq n$. Moreover, let $M(d)$ be the set of monomials of degree $d \geq 0$ in $x_{0}, x_{1}, \ldots, x_{n}$, and

$$
\begin{aligned}
V_{d}^{*} & =\operatorname{Vect}\left(m g_{k}^{*}: m \in M(d+1), \text { and } 0 \leq k \leq n\right), \\
\tilde{V}_{d}^{*} & =\operatorname{Vect}\left(\left\{m \frac{\partial h_{i}^{*}}{\partial x_{j}}: m \in M(d), 0 \leq i \leq u, \text { and } 0 \leq j \leq n\right\}\right) .
\end{aligned}
$$

Algo ${ }_{\mathrm{FDP}}$ returns a solution of FDP (and not Fail) in:

$$
O\left(n^{3(d+3)}\right),
$$

where $d$ is the smallest integer such that $\operatorname{dim}\left(\tilde{V}_{d}^{*}\right) \geq(n+1)|M(d+1)|$.

Proof. Let us suppose that our algorithm returns a solution (and not Fail). According to Corollary 1, we know that for all $i, 0 \leq i \leq n, g_{i}^{*} \in \partial \mathcal{I}_{h}^{*}:\left(x_{n}^{d+1}\right)$. The complexity of AlgoFDP is then dominated by the cost of computing a reduced DRL Gröbner basis $G$ of $\partial \mathcal{I}_{h}^{*}:\left(x_{n}^{d+1}\right)$. This step can be done as explained 
in Section 2. However, an alternative method can be used in this particular situation. This is due to the particular role of $x_{n}$ in a DRL order. From Lemma 1 we know that if $x_{n}^{d+1}$ divides the leading monomial of a polynomial, then it also divides the entire polynomial. Thus, we can restrict our attention to polynomials of a DRL Gröbner Bases $G^{\prime}$ of $\partial \mathcal{I}_{h}^{*}$ whose leading monomials contain $x_{n}^{d+1}$. One can see directly that:

$$
(g \in G: \operatorname{deg}(g)=2)=\left(\frac{g^{\prime}}{x_{n}^{d+1}}: g^{\prime} \in G^{\prime}, \text { and } x_{n}^{d+1} \mid \operatorname{LM}\left(g^{\prime}, \prec_{D R L}\right)\right) .
$$

More precisely, it is sufficient to compute a reduced $(d+3)$-DRL Gröbner basis of $\partial \mathcal{I}_{h}^{*}$. According to Appendix A, this can be done with the $\mathrm{F}_{5}$ algorithm in $O\left(n^{3(d+3)}\right)$. From a practical point of view, the two methods proposed for computing $\mathrm{G}$ are similar. But the last one is more suitable for evaluating the complexity.

Remark 3. It should be noticed that our algorithm can easily be adapted for polynomials $f$ of degree greater that 2 .

\section{Comparison with Previous Approach}

In short, our method can be viewed as a generalization of the approach of D.F. Ye, Z.D. Dai, and K.Y. Lam 25/26. When $u=n$, it is sufficient to consider the ideal $\partial \mathcal{I}_{h}^{*}:\left(x_{n}^{1}\right)$ for recovering $\mathcal{L}(g)$. This is a simplified description of the method described in [25]26]. When $u<n, \partial \mathcal{I}_{h}^{*}:\left(x_{n}^{1}\right)$ no longer provides enough information for recovering $\mathcal{L}(g)$. To overcome this difficulty, we proposed here to consider ideals of the form $\partial \mathcal{I}_{h}^{*}:\left(x_{n}^{d+1}\right)$. We then proved that $\mathcal{L}(g)$ is contained in this ideal as soon as $d$ is sufficiently large.

It is important to know the exact value of the parameter $d$. This value can be lower-bounded in fonction of the ratio $n / u$. For this, we observe that $(n+$ 1) $|M(d+1)|=(n+1) \mathrm{C}_{n+1+d}^{d+1}$ and $\operatorname{dim}\left(\tilde{V}_{d}^{*}\right)$ is very likely to be equal $(u+1)(n+$ 1) $\mathrm{C}_{n+d}^{d}$. We then obtain that $d$ should verify:

$$
d \geq \frac{n}{u}-1 .
$$

For instance, if the number of equations removed (i.e. $n-u$ ) is smaller than $\left\lfloor\frac{n}{2}\right\rfloor$, this yields a complexity of $O\left(n^{12}\right)$, and $O\left(n^{9}\right)$ if $u=n$. We will show now that this approximation is perfectly coherent with our experimental results.

\subsection{Experimental Results}

\section{Generation of the Instances}

We have only considered instances $h=f \circ g$ of FDP admitting a solution. We constructed these instances in the following way:

$-f=t \circ \psi \circ s$ and $g=\phi \circ r$, with $r, s, t \circ \psi \circ s: K^{n} \rightarrow K^{n}$ are random affine bijections, and $\psi, \phi: K^{n} \rightarrow K^{n}$ are S-box functions contructed as explained in Section 3 . We then remove $r \geq 0$ polynomials of $h$. 


\section{Programming language - Workstation}

The experimental results have been obtained with a Xeon bi-processor $3.2 \mathrm{Ghz}$, with $6 \mathrm{~Gb}$ of Ram. The instances of FDP have been generated using the Maple software. We used our own implementation (in language $\mathrm{C}$ ) of $\mathrm{F}_{5}$ for computing truncated Gröbner bases.

\section{Table Notations}

The following notations are used in the table below:

$-n$, the number of variables,

$-b$, the number of blocks (as defined in Section 3),

$-n_{i}$, the number of variables in each block (see Section 3),

$-q$, the size of the field,

$-r$, the number of polynomials removed,

$-d_{\text {theo }}=\left\lceil\frac{n}{u}-1\right\rceil$, the predicted (see 4.2) value of $d$ for which AlgofDP returns a solution

- $d_{\text {real }}$, the real value of $d$ for which AlgoFDP returns a solution

$-T$, the total time taken by our algorithm,

$-\sqrt{q^{n}}$, the current security bound [18]6] for $2 \mathrm{R}^{-}$schemes.

\section{Practical Results}

Let us now present results obtained with our algorithm.

\begin{tabular}{|c|c|c|c|c|c|c|c|c|}
\hline$n$ & $b$ & $n_{i}$ & $r$ & $q$ & $d_{\text {theo }}$ & $d_{\text {real }}$ & $T$ & $\sqrt{q^{n}}$ \\
\hline 8 & 4 & 2 & 0 & 65521 & 0 & 0 & $0.0 \mathrm{~s}$. & \\
\hline 8 & 4 & 2 & 4 & 65521 & 1 & 1 & $0.0 \mathrm{~s}$. & $\approx 2^{64}$ \\
\hline 8 & 4 & 2 & 5 & 65521 & 2 & 2 & $0.3 \mathrm{~s}$. & $\approx 2^{64}$ \\
\hline 8 & 4 & 2 & 6 & 65521 & 3 & 3 & $1.9 \mathrm{~s}$. & $\approx 2^{64}$ \\
\hline \hline 10 & 5 & 2 & 5 & 65521 & 1 & 1 & $0.2 \mathrm{~s}$. & $\approx 2^{80}$ \\
\hline 10 & 5 & 2 & 6 & 65521 & 2 & 2 & $3.2 \mathrm{~s}$. & $\approx 2^{80}$ \\
\hline 10 & 5 & 2 & 7 & 65521 & 3 & 3 & $21.4 \mathrm{~s}$. & $\approx 2^{80}$ \\
\hline 10 & 5 & 2 & 8 & 65521 & 4 & 4 & $180.8 \mathrm{~s}$. & $\approx 2^{80}$ \\
\hline \hline 12 & 3 & 4 & 0 & 65521 & 1 & 1 & $0.1 \mathrm{~s}$. & \\
\hline 12 & 3 & 4 & 5 & 65521 & 1 & 1 & $0.9 \mathrm{~s}$. & $\approx 2^{96}$ \\
\hline 12 & 3 & 4 & 6 & 65521 & 1 & 1 & $0.9 \mathrm{~s}$. & $\approx 2^{96}$ \\
\hline 12 & 3 & 4 & 7 & 65521 & 2 & 2 & $20.5 \mathrm{~s}$. & $\approx 2^{96}$ \\
\hline 12 & 3 & 4 & 8 & 65521 & 2 & 2 & $25.2 \mathrm{~s}$. & $\approx 2^{96}$ \\
\hline 12 & 3 & 4 & 9 & 65521 & 3 & 3 & $414 \mathrm{~s}$. & $\approx 2^{96}$ \\
\hline \hline 20 & 5 & 4 & 0 & 65521 & 0 & 0 & $1.6 \mathrm{~s}$. & \\
\hline 20 & 5 & 4 & 5 & 65521 & 1 & 1 & $55.2 \mathrm{~s}$. & $\approx 2^{160}$ \\
\hline 20 & 5 & 4 & 10 & 65521 & 1 & 1 & $78.9 \mathrm{~s}$. & $\approx 2^{160}$ \\
\hline 20 & 10 & 2 & 10 & 65521 & 1 & 1 & $78.8 \mathrm{~s}$. & $\approx 2^{160}$ \\
\hline 20 & 2 & 10 & 10 & 65521 & 1 & 1 & $78.7 \mathrm{~s}$. & $\approx 2^{160}$ \\
\hline 24 & 6 & 4 & 0 & 65521 & 0 & 0 & $4.9 \mathrm{~s}$. & \\
\hline 24 & 6 & 4 & 12 & 65521 & 1 & 1 & $376.1 \mathrm{~s}$. & $\approx 2^{192}$ \\
\hline
\end{tabular}




\begin{tabular}{|c|c|c|c|c|c|c|c|c|}
\hline 30 & 15 & 2 & 15 & 65521 & 1 & 1 & 2910.5 s. & $\approx 2^{160}$ \\
\hline 32 & 8 & 4 & 0 & 65521 & 0 & 0 & 31.3 s. & \\
\hline 32 & 8 & 4 & 10 & 65521 & 1 & 1 & 3287.9 s. & $\approx 2^{256}$ \\
\hline 32 & 8 & 4 & 16 & 65521 & 1 & 1 & 4667.9 s. & $\approx 2^{256}$ \\
\hline 36 & 18 & 2 & 15 & 65521 & 1 & 1 & 13427.4 s. & $\approx 2^{256}$ \\
\hline
\end{tabular}

\section{Interpretation of the Results}

Let us mention that $n=16$ and $n=32$ were two challenges proposed by the designers of $2 \mathrm{R}^{-}$schemes. First it should be observed that the parameters $b$ and $n_{i}$ of the S-box functions seem irrelevant for the complexity of our algorithm. We also tested our algorithm for instances of FDP constructed with various forms of $\psi, \phi\left(C^{*}+\right.$ S-Box functions, Triangular+S-Box functions, $\left.\ldots\right)$ and several values of $q$. These results are very similar to the ones obtained for S-Box functions, and thus not quoted here. The major observation is that our algorithm behaves exactly as predicted. That is, $d_{\text {theo }}=\left\lceil\frac{n}{u}-1\right\rceil$ is exactly equal to the $d_{\text {real }}$ observed in practice.

\section{Acknowledgements}

We thank Lilian Bohy, Jintai Ding and anonymous referees for numerous comments which improved the presentation of the results.

\section{References}

1. A.W. Adams and P. Loustaunau. An Introduction to Gröbner Bases. Graduate Studies in Mathematics, Vol. 3, AMS, 1994.

2. G. Ars, J.-C. Faugère, H. Imai, M. Kawazoe, and M. Sugita. Comparison Between $X L$ and Gröbner Basis Algorithms. Advances in Cryptology - ASIACRYPT 2004, Lecture Notes in Computer Science, vol. 3329, pp. 338-353, 2004.

3. G. Ars, and J.-C. Faugère. Algebraic Immunities of functions over finite fields. Proceedings of BFCA'05, Rouen, 2005. Also available at http://eprint.iacr. org/2004/188.ps

4. M. Bardet, J-C. Faugère, B. Salvy and B-Y. Yang. Asymptotic Behaviour of the Degree of Regularity of Semi-Regular Polynomial Systems. In MEGA 2005, Eighth International Symposium on Effective Methods in Algebraic Geometry, 15 pages, 2005.

5. M. Bardet, J-C. Faugère, and B. Salvy. On the Complexity of Gröbner Basis Computation of Semi-Regular Overdetermined Algebraic Equations. In Proc. of International Conference on Polynomial System Solving (ICPSS), pp. 71-75, 2004.

6. E. Biham. Cryptanalysis of Patarin's 2-Round Public Key System with S Boxes (2R). Advances in Cryptology - CRYPTO 2000, Lecture Notes in Computer Science, vol. 1807, Springer-Verlag, pp. 408-416, 2000.

7. B. Buchberger. Gröbner Bases : an Algorithmic Method in Polynomial Ideal Theory. Recent trends in multidimensional systems theory. Reider ed. Bose, 1985.

8. B. Buchberger, G.-E. Collins, and R. Loos. Computer Algebra Symbolic and Algebraic Computation. Springer-Verlag, second edition, 1982. 
9. V. Carlier, H. Chabanne, and E. Dottax Grey Box Implementation of Block Ciphers Preserving the Confidentiality of their Design. Proceedings of BFCA'05, Rouen, 2005. Also available at http://eprint.iacr.org/2004/188.ps.

10. N. Courtois, L. Goubin, and J. Patarin. SFLASH, a Fast Asymmetric Signature Scheme for low-cost Smartcards - Primitive Specification and Supporting Documentation. Available at http://www.minrank.org/sflash-b-v2.pdf.

11. D. A. Cox, J.B. Little, and D. O'Shea. Ideals, Varieties, and Algorithms: an Introduction to Computational Algebraic Geometry and Commutative Algebra. Undergraduate Texts in Mathematics. Springer-Verlag. New York, 1992.

12. M. Dickerson. The functional Decomposition of Polynomials. Ph.D Thesis, TR 89-1023, Departement of Computer Science, Cornell University, Ithaca, NY, July 1989.

13. J.-C. Faugère. A New Efficient Algorithm for Computing Gröbner Casis without Reduction to Zero: $\mathrm{F}_{5}$. Proceedings of ISSAC, pp. 75-83. ACM press, July 2002.

14. J. C. Faugère, P. Gianni, D. Lazard, and T. Mora. Efficient Computation of ZeroDimensional Gröbner Bases by Change of Ordering. Journal of Symbolic Computation, 16(4), pp. 329-344, 1993.

15. D. Kozen, and S. Landau. Polynomial decomposition algorithms. J. Symb. Comput. (7), pp 445-456, 1989.

16. L. Goubin, and J. Patarin. Trapdoor One-way Permutations and Multivariate Polynomials. Information and Communication Security, First International Conference (ICICS'97), Lecture Notes in Computer Science vol. 1334, Springer-Verlag, pp. 356-368, 1997.

17. L. Goubin, and J. Patarin. Asymmetric Cryptography with S-Boxes. Information and Communication Security, First International Conference (ICICS'97), Lecture Notes in Computer Science vol. 1334, Springer-Verlag, pp. 369-380, 1997.

18. L. Goubin, and J. Patarin. Asymmetric Cryptography with S-Boxes - Extended Version. Available at http://citeseer.ist.psu.edu/patarin97asymmetric.html.

19. J. Gutierrez, R. Rubio, J. von zur Gathen. Multivariate Polynomial Decomposition. Algebra in Engineering, Communication and Computing, 14 (1), pp. 11-31.

20. T. Matsumoto, and H. Imai. Algebraic Methods for Constructing Asymmetric Cryptosystems. Algebraic and Error-Correcting Codes. Prod. Third Intern. Conf., Grenoble, France, Springer-Verlag, pp. 108-119, 1985.

21. T. Matsumoto, and H. Imai. Public Quadratic Polynomial-tuples for efficient signature-verification and message-encryption. Advances in Cryptology - EUROCRYPT 1988, Lecture Notes in Computer Science, vol. 330, Springer-Verlag, pp. 419-453, 1988.

22. J. Patarin. Cryptanalysis of the Matsumoto and Imai Public Key Scheme of Eurocrypt'88. Advances in Cryptology - CRYPTO 1995, Lecture Notes in Computer Science, Springer-Verlag, vol. 963, pp. 248-261, 1995.

23. J. von zur Gathen. Functional decomposition of polynomials: the tame case. J. Symb. Comput. (9), pp. 281-299, 1990.

24. J. von zur Gathen. Functional decomposition of polynomials: the wild case. J. Symb. Comput. (10), pp. 437-452, 1990.

25. D.F. Ye, K.Y. Lam, Z.D. Dai. Cryptanalysis of "2R" Schemes, Advances in Cryptology - CRYPTO 1999, Lecture Notes in Computer Science, vol. 1666, SpringerVerlag, pp. 315-325, 1999.

26. D.F. Ye, Z.D. Dai and K.Y. Lam. Decomposing Attacks on Asymmetric Cryptography Based on Mapping Compositions, Journal of Cryptology (14), pp. 137-150, 2001. 


\section{Appendix A}

\section{The $\mathbf{F}_{5}$ Algorithm}

The historical method for computing Gröbner bases is Buchberger's algorithm 817. Recently, more efficient algorithms have been proposed. To date, $\mathrm{F}_{5}$ [13] is the most efficient for computing Gröbner bases. In a nutshell, this algorithm constructs incrementally the following matrices in degree $d$ :

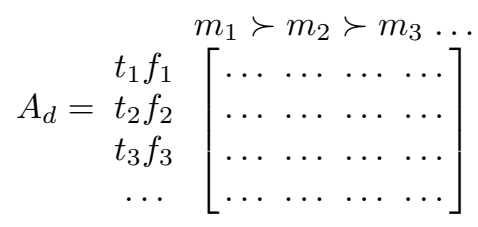

where the indices of the columns are monomials sorted for the admissible ordering $\prec$ and the rows are product of some polynomials $f_{i}$ by some monomials $t_{j}$ such that $\operatorname{deg}\left(t_{j} f_{i}\right) \leq d$. For a regular system [13] the matrices $A_{d}$ are of full rank. In a second step, row echelon forms of theses matrices are computed, i.e.

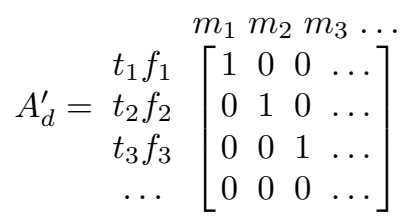

Note that for each $d, A_{d}^{\prime}$ contains a $d$-Gröbner basis of the ideal considered. Important parameters to evaluate the complexity of $F_{5}$ is the maximal degree $d$ occurring in the computation and the size of the matrix $A_{d}$. The overall cost is thus dominated by $\left(\# A_{d}\right)^{3}$. Very roughly, $\left(\# A_{d}\right)$ can be approximated by $O\left(n^{d}\right)$. A more precise complexity analysis can be found in [4/5]. 